\title{
Grain-Boundary Roughening in Colloidal Crystals
}

\author{
Maijia Liao, ${ }^{1}$ Xiao Xiao, ${ }^{1}$ Siu Tat Chui, ${ }^{2}$ and Yilong Han ${ }^{1, *}$ \\ ${ }^{1}$ Department of Physics, The Hong Kong University of Science and Technology, Hong Kong, China \\ ${ }^{2}$ Department of Physics and Astronomy, University of Delaware, Newark, Delaware 19716, USA
}

(Received 7 May 2017; revised manuscript received 8 January 2018; published 16 May 2018)

\begin{abstract}
In polycrystals, faceted grains may become round and rough at high temperatures. Such a roughening phenomenon remains poorly understood, partly because of the lack of experimental observations. Here, we directly visualize the roughening dynamics of grain boundaries inside thin-film colloidal crystals at the single-particle level using video microscopy. The thermal fluctuations of grain boundaries appear to exhibit both static and dynamic critical-like behaviors, in contrast to the Kosterlitz-Thouless transition in typical free surface roughening. The roughening point shifts towards the melting point as the grain boundary's mismatch angle $\theta$ decreases and is preempted by melting when $\theta<18^{\circ}$. Counterintuitively, the amplitude of grain-boundary fluctuations decreases above the roughening point. This could be attributed to the observed widening of the grain boundary. The roughening strongly affects the mobility of the grain boundary but not the stiffness. These results provide new guidance for the control of microstructures in polycrystals and further development of roughening theory.
\end{abstract}

DOI: 10.1103/PhysRevX.8.021045

\section{INTRODUCTION}

Grain boundaries are the longest known but least understood crystal defects [1]. They have significant effects on the properties of a broad class of polycrystalline materials, including metals, alloys, ceramics, minerals, magnets, and semiconductors [2]. The understanding and proper control of grain boundaries are key to grain growth and stagnation [3], superplasticity [4], recrystallization [5], corrosion, fracture, and the transport properties of high $T_{c}$ superconductors [6], and they continue to be major goals in material science and engineering. The roughening transition, representing a dramatic change in the grain boundary, is crucial to microstructure evolutions [3] but has rarely been explored.

Polycrystalline grains are usually faceted because some of the grain boundaries with certain angles have lower interfacial energy $U$ than others. As temperature $T$ increases, the entropy $S$ becomes more important in the free energy of the grain boundary, $F=U-T S$. When the free energy cost of creating a small step on the grain boundary decreases to zero, it triggers the proliferation of

\footnotetext{
* Corresponding author. yilong@ust.hk

Published by the American Physical Society under the terms of the Creative Commons Attribution 4.0 International license. Further distribution of this work must maintain attribution to the author(s) and the published article's title, journal citation, and DOI.
}

Subject Areas: Materials Science, Soft Matter,

Statistical Physics steps to form a completely rough and disordered interface with high entropy. This mechanism of thermal roughening generally applies to various interfaces [2], but the roughening transition mainly refers to the roughening of a crystal's free surface, i.e., solid-vapor interface [7]. Such surface roughening has been theoretically predicted to follow a KosterlitzThouless (KT) transition [8,9] and has been experimentally studied using electron or optical microscopy [10-12].

Grain boundaries can also exhibit thermal roughening, but the phenomenon has only been measured for surface grain boundaries, i.e., thermal grooves [2], which are onedimensional (1D) intersections between a free surface and a bulk grain boundary in a three-dimensional (3D) crystal. A polycrystal contains numerous buried grain boundaries but only several faceted surfaces; therefore, grain-boundary roughening should affect more material properties than surface roughening and surface grain-boundary roughening. The roughening transition theory applies to free surfaces without strain [8,13-15], but a grain boundary is sandwiched between two anisotropic lattices with complex structures and possible strain. The broad length scales and timescales in grain-boundary roughening pose challenges to simulations and experiments. To speed up the sluggish dynamics in solids, external driving forces were usually employed in simulations [16-18] and experiments $[19,20]$. The resulting kinetic roughening under a nonequilibrium drive is generally considered as a crossover rather than a genuine phase transition [21]. Moreover, bulk grain boundaries are difficult to control and measure in situ. Theoretical modeling grain-boundary roughening has been 
briefly discussed for low-angle grain boundaries [15,22] but not yet for high-angle grain boundaries because of the complicated elastic energy. A grain boundary depends on the interference of two crystalline lattices, which is more complex than a free surface [23]. Thus, it is possible that grain-boundary roughening is not a KT transition. Consequently, many open questions exist: How is the grain-boundary roughening different from surface roughening? How does the roughening affect the grain-boundary properties such as stiffness, mobility, and the melting behavior?

Motivated by these open questions, our experiments seek to observe the roughening transitions of grain boundaries buried in colloid crystals. This class of model systems, composed of micrometer-sized colloidal particles whose thermal motion can be directly visualized using video microscopy, have already provided many general insights about phase transitions in bulk, including crystallization, melting, sublimation, and solid-solid, glass, and jamming transitions [24]. Grain-boundary dynamics [25] and kinetic roughening [19] at a fixed volume fraction have been explored in colloidal crystals, but thermal roughening phase transitions have not. Here, we employ tunable colloids to study the thermal roughening phase transition of buried grain boundaries with single-particle dynamics.

\section{MATERIALS AND METHODS}

This colloid was absorbed into a 10- $\mu$ m-thick parallelplate cell by the capillary force. The flow during this process assembled N-isopropylacrylamide (NIPA) spheres into a face-centred cubic (fcc) crystal with its (111) plane parallel to the plates. The polycrystal can be melted, recrystallized, and annealed to the desired average grain size. We cycle the temperature near the melting point of the crystal to anneal some of the defects away and to release any stress. We use thin-film crystals with a thickness of 15-20 layers, and thus all grain boundaries were ribbonlike with a uniform shape along the $z$ direction and perpendicular to the parallel plates [Fig. 1(a)]. Because the refractive index of the NIPA spheres is very close to that of water, a layer in the middle was monitored using brightfield microscopy. We observed the colloidal polycrystals at the mesoscopic scale using Bragg diffraction microscopy [19]. The colloidal sample was illuminated with a beam of white light, and the objective was placed at the first Bragg diffraction spot [19]. Different lattice orientations yield different diffraction colors, and thus a polycrystal is composed of iridescent patches as shown in Figs. 1(b) and 1(c). Grain boundaries are disordered and thus are shown in black in Figs. 1(b) and 1(c). Easily switching between Bragg diffraction microscopy and bright-field microscopy enables observation in broad length scales.

\section{RESULTS}

\section{A. Power-spectrum analysis of grain-boundary shape}

Our thin-film colloidal crystals are composed of 15 layers of poly NIPA spheres confined in glass cells [Fig. 1(a)]. The effective diameter $\sigma$ of the NIPA sphere
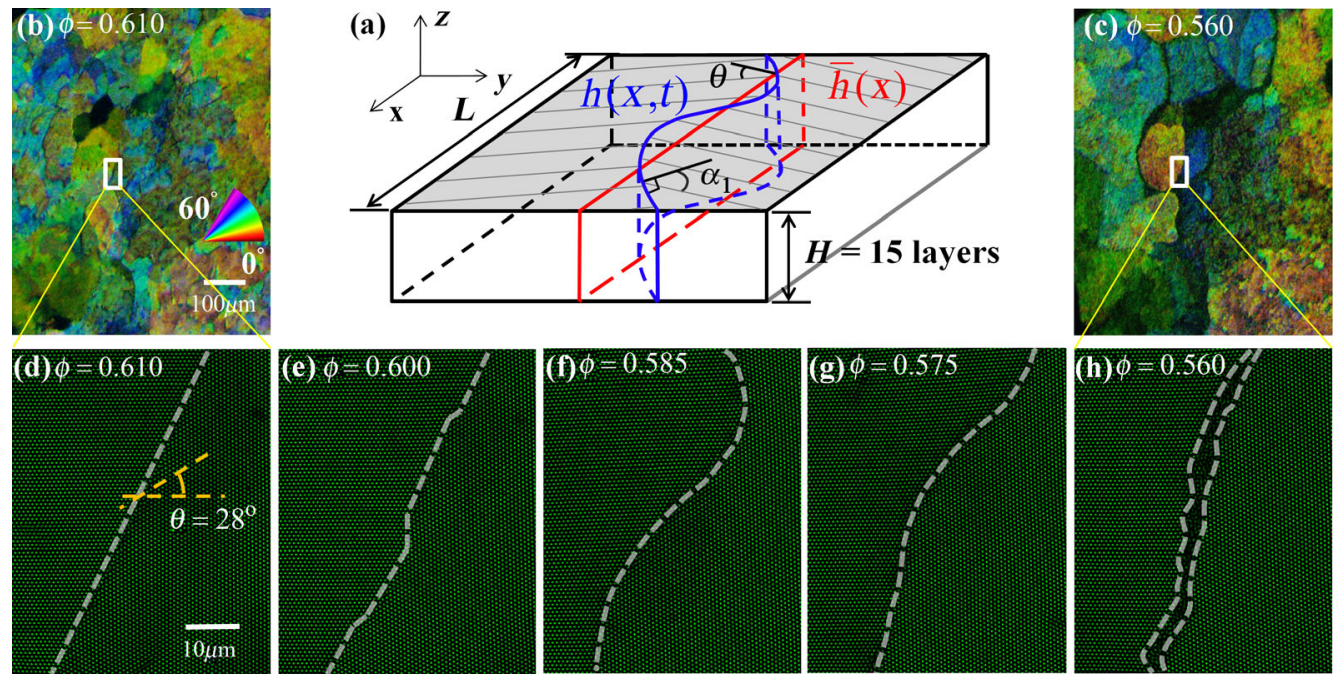

FIG. 1. Evolution of the grain-boundary morphology. (a) Schematic illustration of a grain boundary in a thin-film colloidal crystal with lateral length $L$, mismatch angle $\theta$, and an inclination angle $\alpha_{1}$. The shape of the grain boundary $h(x, t)$ (blue curve) is defined as the displacement relative to the average position of the grain boundary $\bar{h}(x)$ (red line). The parallel grey lines in the shaded area represent the [110] direction of the lattices. (b,c) Raw images of the colloidal polycrystal under the Bragg-diffraction microscopy at (b) $\phi=0.610$ and (c) $\phi=0.560$. The true colors represent the local $[110]$ lattice orientation $\beta \in\left[0^{\circ}, 60^{\circ}\right]$ shown in (b). Grains with $\beta=0^{\circ}$ and disordered grain boundaries are shown in black. (d)-(h) The grain boundary with $\theta=28^{\circ}$ in the small rectangular area in panels (b) and (c) is straight in panel (d), develops steps in panel (e), and is curved and completely rough without line segments in panel (f). Its fluctuation amplitude decreases in panel (g), and finally, it melts in panel (h). 
changes from $0.87 \mu \mathrm{m}$ at $21.9^{\circ} \mathrm{C}$ to $0.70 \mu \mathrm{m}$ at $30.6{ }^{\circ} \mathrm{C}$ in water (Fig. S1 in Ref. [26]). The spheres have short-range repulsive interactions and exhibit almost the same phase behavior as hard spheres $[27,28]$. The colloid flows into the sample cell, which anneals the (111) plane of the fcc polycrystal along the two parallel plates of the cell. The inverse of the volume fraction $\phi^{-1}$ in colloids plays a similar role to effective temperature in atomic systems, and thus decreasing $\phi$ can drive the roughening of the grain boundary and crystal melting. Bulk particles can be clearly imaged using bright-field microscopy [Figs. 1(d)-1(h)] [27]. The grain boundaries are ribbonlike with a uniform shape along the $z$ direction, as shown in Fig. 1(a). We did not measure thick crystals with more than 30 layers because their grain boundaries often become curved along the $z$ direction, which makes the dynamics of the whole grain boundary difficult to monitor. Different lattice orientations in the polycrystal exhibit different colors in Bragg diffraction microscopy [19] [see Figs. 1(b) and 1(c) and Supplemental Material (SM) in Ref. [26]]. The sample temperature is increased in steps of $0.1^{\circ} \mathrm{C}$ (i.e., $\phi$ decreased in steps of $0.4 \%$ ) until the grain boundary melts. After full equilibration at each step, the thermal fluctuations of the grain boundary are recorded for $1 \mathrm{~h}$ using a charge-coupled device camera at 2 frames/s. Particle positions are then determined from the image analysis [29].

In quasi-2D crystals, a grain boundary is characterized by three angles [30]: the mismatch angle $\theta$ between the orientations of two adjacent lattices, and the inclination angles $\alpha_{1}, \alpha_{2}$ between the normal direction of the grain boundary and the orientation of each lattice [Fig. 1(a)]. For a triangular lattice, $\alpha_{1}+\alpha_{2}=60^{\circ}-\theta$; hence, two of the three angles are independent. When the grain boundary fluctuates, $\theta$ is a constant while $\alpha$ changes. As $T$ increases (i.e., $\phi$ decreases), the flat grain boundary with $\theta=28^{\circ}$, $\alpha_{1}=28^{\circ}$, and $\alpha_{2}=4^{\circ}$ in Fig. 1(d) first develops small steps in a defaceting process [Fig. 1(e)], is completely rough without line segments at the roughening point $\phi_{\mathrm{R}}=0.585$ [Fig. 1(f)], and eventually melts [Fig. 1(h)]. Counterintuitively, the amplitude of the grain-boundary oscillation decreases after the roughening [Fig. 1(g)].

The shape of the grain boundary at time $t$ is described by the height function $h(x, t)$, i.e., the displacement from the average position $\bar{h}(x)$ of the grain boundary over the observation period of about $1 \mathrm{~h}$ [see Fig. 1(a)]. The morphology and dynamics of the grain boundary are quantified by the power spectra [Fig. 2(a)] and the spatial and time correlations of $h(x, t)$ [Figs. 2(b) and 2(c)]. We fit (a)

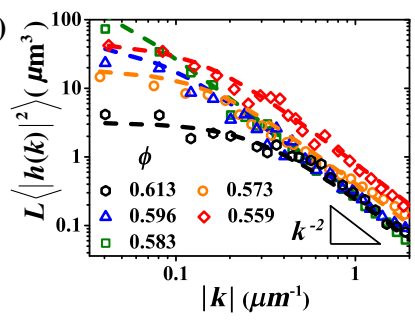

(d)

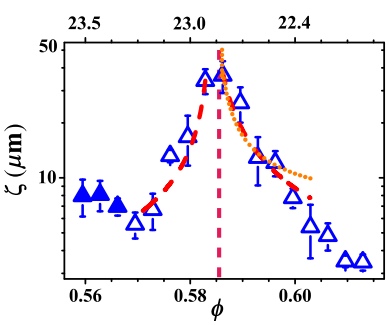

(b)

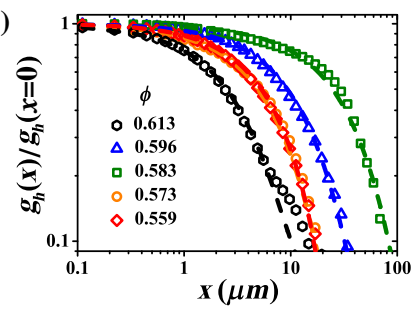

(e)

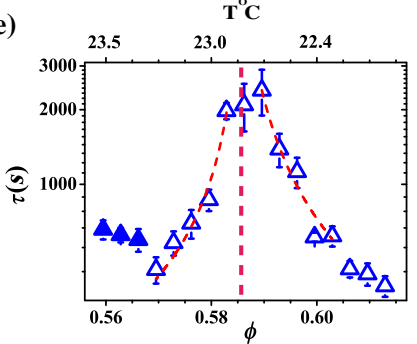

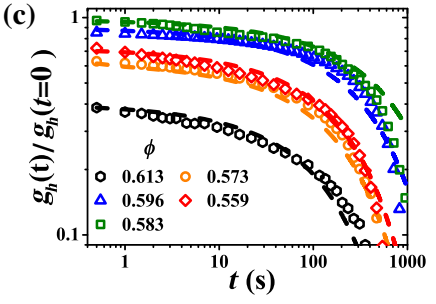

(f)

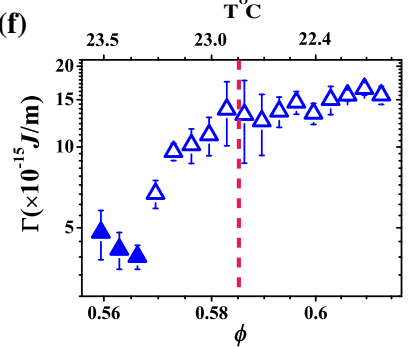

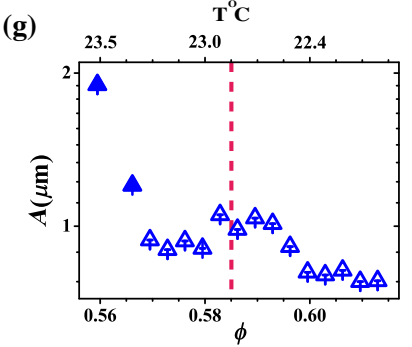

FIG. 2. Critical behaviors and properties of a high-angle $\left(\theta=28^{\circ}\right)$ grain boundary (HAGB). (a) Power spectra of the height function (symbols) fitted with Eq. (1) (dashed curves) at different volume fractions. (b) Normalized spatial correlation functions $g_{h}(x) / g_{h}(0)$ (symbols) fitted with $\exp (-x / \xi)$ (dashed curves). (c) The normalized time correlation $g_{h}(t) / g_{h}(0)$ (symbols) fitted with erfc $(\sqrt{t / \tau})$ (dashed curves). (d) Correlation lengths fitted with $\xi=\xi_{0} s^{-\nu}$ (red dashed curves), where $s=\left(\phi^{-1}-\phi_{\mathrm{R}}^{-1}\right) / \phi_{\mathrm{R}}^{-1}$ is the renormalized temperature. The fitted $\phi_{\mathrm{R} 1}=0.584, \xi_{1}=0.65 \pm 0.36 \mu \mathrm{m}$, and $\nu_{1}=0.74 \pm 0.11$ for the preroughening branch and $\phi_{R 2}=0.584$, $\xi_{2}=0.87 \pm 0.33 \mu \mathrm{m}$, and $\nu_{2}=0.59 \pm 0.07$ for the postroughening branch. The data deviate from the KT prediction $\xi \sim$ $\exp \left[B / \sqrt{\left(1 / \phi_{\mathrm{R}}-1 / \phi\right)}\right]$ (orange dashed curve) and $\phi_{\mathrm{R}}=0.584$, and can be clearly confirmed by the nonlinear behavior of $\xi \sqrt{\left|1 / \phi_{R}-1 / \phi\right|}$ in Fig. S2 (Ref. [26]). (e) The correlation time fitted with $\tau=\tau_{0} s^{-\nu z}$ (red dashed curves), where $\tau_{1}=10.4 \pm 4.5 \mathrm{~s}$, $z_{1}=1.58 \pm 0.25$, and $\phi_{\mathrm{R} 1}=0.584$ for the preroughening branch and $\tau_{2}=16.1 \pm 5.7 \mathrm{~s}, z_{2}=1.55 \pm 0.19$, and $\phi_{\mathrm{R} 2}=0.586$ for the postroughening branch. (f) The stiffness $\Gamma$ extracted from Eq. (1) with $\xi$ fitted from panel (d). (g) The fluctuation amplitude $A$ of the grain boundary. Filled symbols in panels $(\mathrm{d})-(\mathrm{g})$ represent the properties of the two solid-liquid interfaces of the melted grain boundary. The error bars in panels (d)-(g) and in Fig. 4 were estimated using ten slightly different baselines, which have less than $5^{\circ}$ angle deviations from the averaged baseline. 
the power spectra with the following equation from capillary wave theory $[19,25]$ :

$$
\left\langle|h(k)|^{2}\right\rangle=\frac{k_{\mathrm{B}} T}{L \Gamma\left(k^{2}+\xi^{-2}\right)},
$$

where $k_{\mathrm{B}}$ is the Boltzmann constant, $L$ is the lateral length shown in Fig. 1(a), and $\Gamma$ is the stiffness of the grain boundary. At large wave vector $k$, the power spectrum is proportional to $k^{-2}$ as capillary waves between two liquid interfaces. The deviation from $k^{-2}$ at a small $k$, i.e., in the long wavelength regime, can be attributed to a cutoff length $\xi[19,25]$. Equation (1) is used to describe colloidal crystalliquid interfaces [31,32], liquid-gas interfaces [33], and grain boundaries [19,25]. From Eq. (1), the normalized spatial correlation function can be derived as $g_{\mathrm{h}}(x)=$ $\left\langle h\left(x_{0}+x, t_{0}\right) h\left(x_{0}, t_{0}\right)\right\rangle_{x_{0}, t_{0}}=g_{\mathrm{h}}(0) \exp (-x / \xi) \quad$ (see SM [26]) [19,25], where \langle\rangle$_{x_{0}, t_{0}}$ is the average over all positions $x_{0}$ and all times $t_{0}$. Note that $\xi$ reflects the correlation length. The decay of $g_{\mathrm{h}}(x)$ is slowest at $\phi_{\mathrm{R}}$ [Fig. 2(b)], hence the fitted $\xi$ peaks at $\phi_{\mathrm{R}}$ [Fig. 2(d)].

\section{B. Correlation functions of grain boundaries}

The grain-boundary dynamics can be characterized by the time autocorrelation function in real space $g_{h}(t)=$ $\left\langle h\left(x_{0}, t_{0}\right) h\left(x_{0}, t_{0}+t\right)\right\rangle_{x_{0}, t_{0}}$ or in Fourier space $S(k, t)=$ $\langle h(k, 0) h(k, t)\rangle$. We assume that $S(k, t)$ is related to the power spectrum in the usual way as $S(k, t)=$ $\langle h(k, 0) h(k, t)\rangle=\left\langle|h(k)|^{2}\right\rangle \exp \left(-M \Gamma k^{2} t\right)$, where $M$ is the renormalized mobility of the grain boundary [34], which we extract from experimental data. The Fourier transform of $S(k, t)$ yields $g_{h}(t) / g_{h}(0)=\operatorname{erfc}(\sqrt{t / \tau})$, where erfc is the complementary error function and the correlation time $\tau=\xi^{2} /(\Gamma M)$. In Fig. 2(c), $g_{h}(t) / g_{h}(0)$ decreases the most slowly at $\phi_{R}$, and thus the fitted $\tau$ in Fig. 2(e) peaks at $\phi_{R}$. For a mode of wavelength $50 \mu \mathrm{m}$, the relaxation time $\tau_{k}=1 /\left(M \Gamma k^{2}\right)=7 \mathrm{~min}$, which is much less than the approximately 1 -h equilibration time and the subsequent measurement time of about $1 \mathrm{~h}$ at each temperature step. In fact, the sample needs a much shorter equilibration because it had already been close to the equilibrium after a temperature change of merely $0.1{ }^{\circ} \mathrm{C} /$ step (i.e., volume fraction $0.4 \% /$ step).

The correlation lengths in Fig. 2(d) can be reasonably fitted with $\xi=\xi_{0}\left[\left(\phi_{R}-\phi\right) / \phi\right]^{-\nu}$ on both sides of $\phi_{R}$, i.e., corresponding to the critical behavior of a continuous phase transition [35]. Both branches around the peak yield the fitted $\phi_{R}=0.584$, which is in good agreement with the directly observed $\phi_{R}=0.585$ from Fig. 1(f). The exponents for the two branches are $\nu_{1}=0.74 \pm 0.11$ and $\nu_{2}=0.59 \pm 0.07$. Figure 2(c) shows $\tau \sim \xi^{z}$ with $z_{1}=$ $1.58 \pm 0.25$ and $z_{2}=1.55 \pm 0.19$ in Fig. 2(e), indicating a dynamic critical slowing down. The lag plots of displacements in Fig. 8 also confirm the strongest correlation at $\phi_{R}$.
Simulation [36] and theory [37] have suggested that free surface roughening may exhibit critical behavior, but experimental evidence is not available. Here, we observe possible critical behavior in grain-boundary roughening, but we cannot rule out other possibilities such as a noncritical behavior or a crossover peak rather than a sharp transition. This is because our fittings are based on several data points around the peak without spanning several orders of magnitude because of the limited resolution.

The fitted stiffness $\Gamma$ from Eq. (1) decreases closer to the melting point $\phi_{m}$ as expected [Fig. 2(f)]. The roughening does not affect $\Gamma$ much, indicating that $\Gamma$ is not responsible for the strongest fluctuation of the grain boundary at $\phi_{R}$. Figures $2(d)-2(g)$ show that the grain boundary and the solid-liquid interface of the melted grain boundary behave differently. The fluctuation amplitude characterized by $A=\left\langle\left[h(x, t)-\langle h(x, t)\rangle_{x}\right]^{2}\right\rangle_{x, t}^{1 / 2}[36,38]$ in Fig. 2(g) peaks near $\phi_{R}$.

\section{Grain-boundary widening}

The KT transition of the surface roughening predicts $\xi \sim \exp \left[B / \sqrt{\left(T_{R}-T\right)}\right]$ [35] below the roughening temperature $T_{R}$ and divergent $\xi$ and $A$ above $T_{\mathrm{R}}[9,38]$, which is distinct from our observation in Figs. 2(d) and 2(g). Figures $2(\mathrm{~d})$ and $2(\mathrm{~g})$ show that they decrease after the roughening, indicating that the fluctuation becomes weaker, as can be directly observed in Figs. 1(f) and 1(g) and Movie 1 in Ref. [26]. This could be attributed to the observed grainboundary widening at $\phi<\phi_{R}$ [Fig. 3(a)]. The widening of grain boundaries to a few particles thick has been observed in simulations [39] and atomic systems [40] near the melting temperature $T_{m}$, but its impact on roughening is not clear. We measure the sixfold orientational order parameter $\psi_{6 j}=\left(\sum_{k=1}^{N_{n}} e^{6 i \theta_{j k}}\right) / N_{n}$, where $\theta_{j k}$ is the angle of the bond between particle $j$ and its neighbor $k$, and $N_{n}$ is the number of nearest neighbors identified through Delaunay triangulation. We smooth the positions of grain-boundary particles whose $\left|\psi_{6}\right|$ is less than 0.8 and obtain the center of the grain boundary, as indicated by the white curve in Fig. 3(b). The grain-boundary particles exhibit a Gaussian probability distribution along the normal direction of the grain boundary (Fig. S3 in Ref. [26]) whose full width at half maximum, $W$, is defined as the width of the grain boundary. Alternatively, grain-boundary particles can be defined as those particles without six neighbors (Fig. S2c in Ref. [26]). This definition yields similar $W$. We find that $W$ starts to increase after $\phi_{R}$ and grows rapidly as soon as the grain boundary begins melting at $\phi_{m}$, as shown in Fig. 3(a). The melted grain boundary turns into a liquid stripe in which the particles possess highly disordered structures and actively swap positions with each other (Movie 1).

We find that the two crystal-liquid interfaces of a melted grain boundary move cooperatively and are positively correlated (Fig. 9). As an important type of heterogeneous 


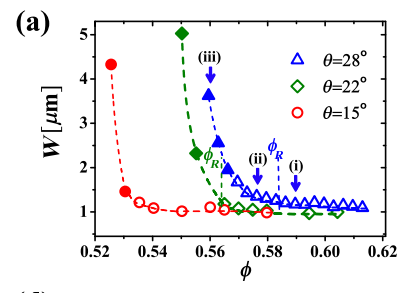

(d)

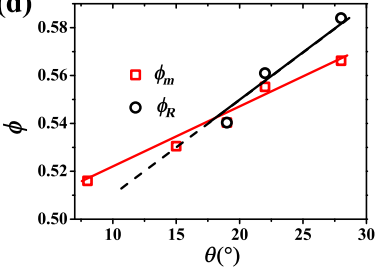

(b)

(i)

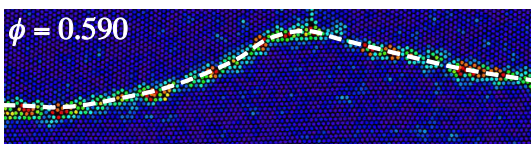

(ii)

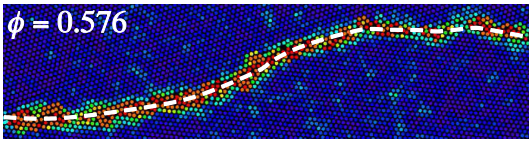

(iii)

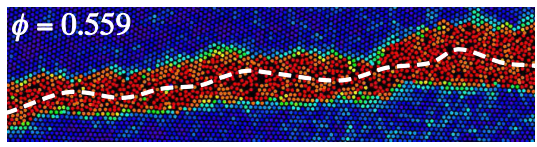

(c)

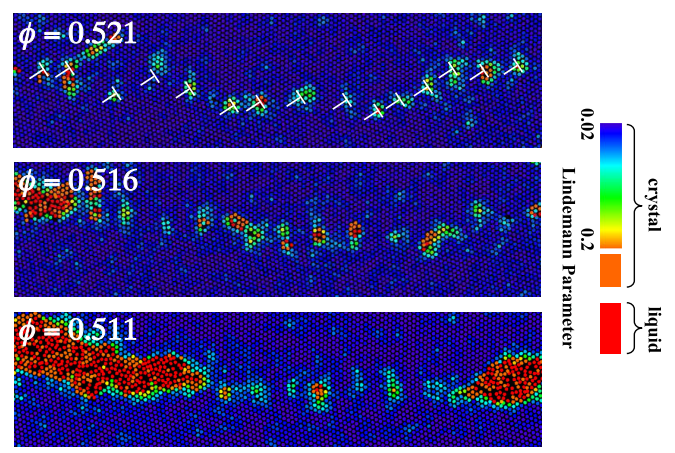

FIG. 3. Grain-boundary widening and melting. (a) Widths of three grain boundaries with $\theta=28^{\circ}, 22^{\circ}$, and $15^{\circ}$. The vertical dashed lines mark the roughening points. (b) The grain boundary with $\theta=28^{\circ}$ colored according to the dynamic Lindemann parameters $L$ (see SM for definition) at (i) preroughening, (ii) postroughening, and (iii) melting labeled in (a) (Movie 1 in Ref. [26]). Liquidlike particles are identified by large dynamic Lindemann parameters $L>0.2$ and low bond-orientational orders $\left|\psi_{6}\right|<0.6$ [41]. The particles keep swapping positions with neighbors (Movie 1) and thus are indeed liquidlike. The white dashed curve represents the center of the grain boundary. (c) The grain boundary with $\theta=8^{\circ}$ is composed of dislocations labeled with $\perp$ (Movie 2 in Ref. [26]). (d) The roughening points and melting points of grain boundaries with different mismatch angles.

melting, grain-boundary melting is difficult to observe inside atomic bulk crystals. It has been observed in colloidal crystals but only for buried high-angle grain boundaries (HAGBs) [27]. HAGBs $\left(\theta>15^{\circ}\right)$ have complex structures, while low-angle grain boundaries (LAGBs) $\left(\theta<15^{\circ}\right)$ can be modeled as an array of separate dislocations. Here, we observe the melting of LAGBs for the first time, which is distinct from the melting of HAGBs. A HAGB directly transforms into a thin liquid layer [Figs. 1(h) and 3(b)] whose thickness increases rapidly as $\phi$ decreases [Fig. 3(a)], signifying a premelting rather than a melting via a nucleation. Premelting is a wetting phenomenon when the interfacial energies satisfy $\gamma_{\text {g.b. }}<$ $2 \gamma_{\text {crystal-liquid }}$ below the bulk melting temperature. It is not a phase transition but a precursor of the melting transition. By contrast, we find that LAGBs first melt into disconnected liquid nuclei from some of their disconnected dislocations before growing and coalescing into a liquid stripe at higher temperatures, as shown in Fig. 3(d). The liquid nucleation at dislocations, rather than forming a wetting layer, is consistent with the small $\gamma_{\text {LAGB }}$ because of the good lattice matching [39]. This melting behavior of buried LAGBs was predicted in simulations [42] but has not been experimentally observed. One- or two-layer liquidlike particles near a dislocation or a planar partial dislocation have been observed in 3D colloidal crystals, but these defects are not strong enough to produce large liquid pockets as $\phi$ decreases [27]. Generally, a higher-angle grain boundary has a higher $\gamma$ [39], and thus its premelting and roughening should occur at a higher $\phi$. This is confirmed in Fig. 3(c). The measured $\phi_{m}(\theta)$ and $\phi_{R}(\theta)$ in Fig. 3(d) are roughly linear, with different slopes, and intersect at $\theta \simeq 18^{\circ}$, suggesting that the roughening is preempted by melting when $\theta \leq 18^{\circ}$. This confirms the theoretical prediction that grain boundaries with a sufficiently small $\theta$ will melt before they have a chance to become rough [22].

\section{Mobility}

The mobility of a grain boundary is important for microstructure evolution and has been a key focus in the study of polycrystalline materials [1]. However, the mobility of a buried grain boundary is sensitive to the segregated impurities; hence, clean grain boundaries have mainly been studied in simulation [1]. Mobility $M=v / F$ is usually measured from the drift speed $v$ under an external driving force $F[19,43]$. Alternatively, it can be measured from the equilibrium thermal diffusion of the center of mass of the grain boundary, $\bar{h}(t)=(1 / L) \int_{0}^{L} h(x, t) d x$, which satisfies $\langle\bar{h}\rangle^{2}=2 D t$ (Fig. S4 in Ref. [26]) and gives $M=D L /\left(k_{B} T\right)$, where $D$ is the diffusion constant [44]. We confirm the 1D random walk of $\bar{h}$ (Fig. S4) as observed in the simulation of 3D metallic polycrystals $[44,45]$. The measured $M(\phi)$ decreases monotonically for the LAGB with $\theta=15^{\circ}$ but nonmonotonically with four distinct regimes for the HAGB with $\theta=28^{\circ}$, as shown in Fig. 4. Before the grain-boundary melting, the mobility $M$ exhibits three regimes: $M(\phi)$ decreases slowly in regime $1(\phi>0.603)$ and rapidly in regime 2 $(0.603<\phi<0.589)$. In regime 3 (0.589<申<0.569), near the melting point, $M(\phi)$ increases. The nonmonotonicity and the peaks of $\xi, \tau$, and $M$ at the roughening point have been confirmed in another experiment involving a 22layer crystal. Such nonmonotonic $M$ in thin-film colloidal crystals is different from the typical simulation behaviors of the grain boundaries in many 3D atomic crystals in which $M$ increases monotonically with $T$ [16]. Nevertheless, 


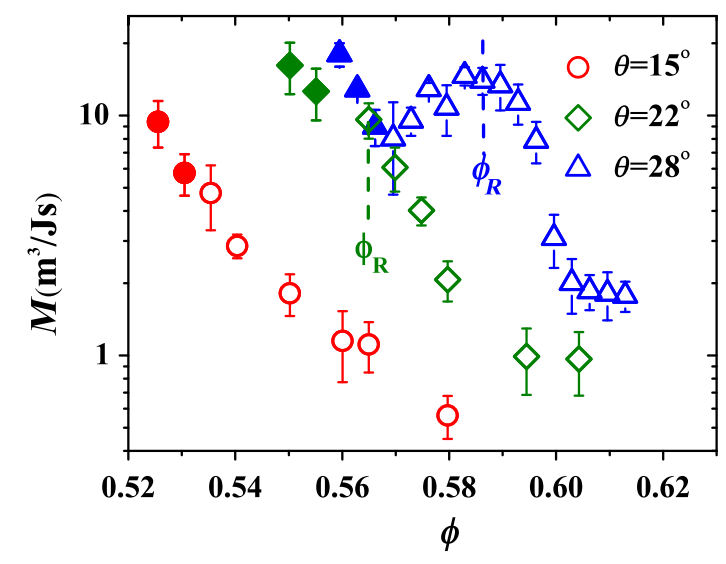

FIG. 4. Mobility $M$ of three grain boundaries with $\theta=28^{\circ}, 22^{\circ}$, and $15^{\circ}$. Solid symbols represent solid-liquid interfaces after melting. Here, $\phi_{\mathrm{R}}$ is marked by vertical dashed lines.

similar nonmonotonic $M$ has been observed in the $\Sigma 7$ grain boundary of AL near the melting point $[18,45]$; this unusual phenomenon was attributed to the possible structural change of the grain boundary $[18,45]$. Here, we further suggest that the structure change could be the widening of the grain boundary, but this conjecture needs to be tested. The maximum $M$ corresponds to the roughening point $\phi_{R}$ in our system, but a roughening point was not reported in the AL system [45]. Nevertheless, the colloidal thin films and 3D atomic crystals could exhibit different behaviors, which is worth further exploration. For the grain boundary with $\theta=22^{\circ}$, the abnormal regime vanishes because $\phi_{R} \simeq \phi_{m}$ (Fig. 5).

\section{DIMENSIONALITY EFFECT}

Dimensionality strongly affects crystal properties and melting behaviors, but its effect on roughening is not clear. We observe that grain boundaries cannot be as easily roughened in 3D colloidal crystals as in thin films, while HAGBs in monolayer colloidal crystals are always rough [46] because lower-dimensional crystals are much softer, with stronger long-wavelength fluctuations [24]. Note that 2D is the critical dimension [47]; hence, even bilayers have different behaviors from monolayers, e.g., in premelting and melting $[48,49]$. The top and bottom walls help us to form ribbonlike grain boundaries with uniform shape in the $z$ direction, but the fluctuations in the $x y$ plane are much weaker in a 20-layer crystal than in a monolayer crystal. This can be understood from a simple lattice-spring model [50]: A 2D square lattice has half of its springs connected in series and half connected in parallel; a two-layer square lattice has $2 / 5$ of its springs in series and $3 / 5$ in parallel; and a $3 \mathrm{D}$ cubic lattice has $1 / 3$ of its springs in series and $2 / 3$ in parallel. A higher fraction of springs in series corresponds to a softer structure. More springs connected in series results in softer structures. The rigidity of a 20-layer crystal is close to that of a 3D crystal; hence, its grain boundary can remain flat when the thermal fluctuation is weak at low effective temperatures, i.e., high packing fractions. Moreover, there are inevitably some small fluctuations with an amplitude of a few particles in the $z$ direction, which makes our system not perfectly $2 \mathrm{D}$, i.e., slightly above the critical dimension. Hence, grain boundaries in the 20-layer crystals can exhibit roughening.

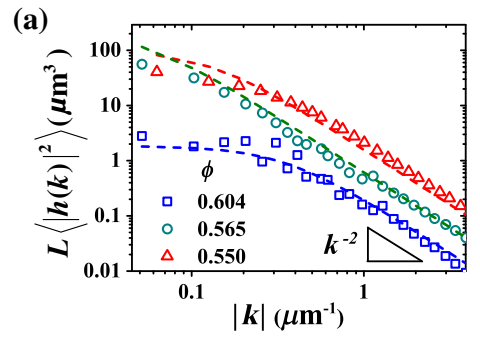

(d)

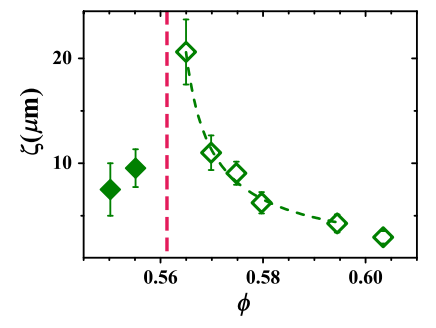

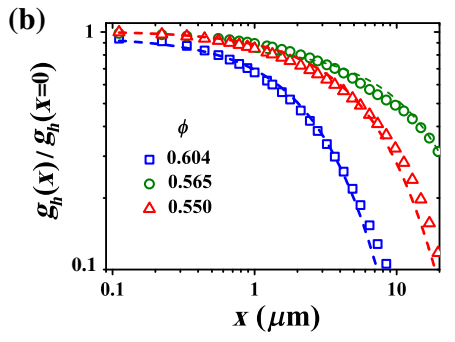

(e)

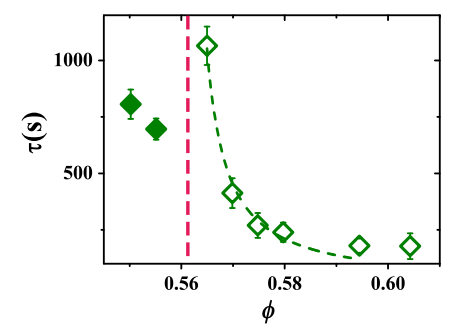

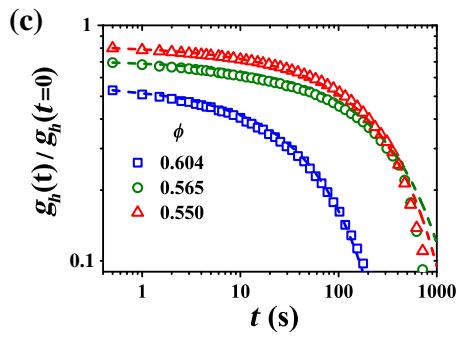

(f)

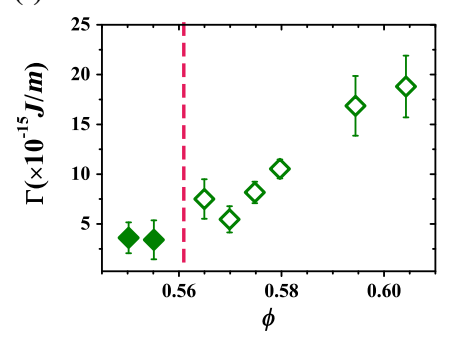

FIG. 5. Correlations and stiffness of the grain boundary with a mismatch angle $\theta=22^{\circ}$ at different volume fractions. (a) Power spectra fitted with Eq. (A3) (dashed curves). (b) The normalized spatial correlation $g_{h}(x) / g_{h}(0)$ fitted with $\exp (-x / \xi)$. (c) The normalized time correlation $g_{h}(t) / g_{h}(0)$ fitted with $\operatorname{erfc}(\sqrt{t / \tau})$. (d) The correlation length fitted with $\xi=\xi_{0}\left|\left[\left(\phi_{R}-\phi\right) / \phi\right]\right|^{-\nu}$, where $\nu=0.74 \pm 0.04$, $\phi_{R}=0.561$, and $\xi_{0}=0.52 \pm 0.08 \mu \mathrm{m}$. (e) The relaxation time fitted with $\tau=\tau_{0}\left|\left[\left(\phi_{\mathrm{R}}-\phi\right) / \phi\right]\right|^{-\nu z}$, where $z=1.4 \pm 0.2, \phi_{R}=0.561$, and $\tau_{0}=5.9 \pm 2.3 \mathrm{~s}$. (f) The stiffness obtained from the fitting in panel (a). Filled symbols in panels (d)-(f) represent the properties of two solid-liquid interfaces of the melted grain boundary. 


\section{CONCLUSION}

In summary, we observed the roughening of buried grain boundaries for the first time using tunable colloidal crystals. The morphologies and dynamics of grain boundaries and melted crystal-liquid interfaces were measured at the single-particle level, revealing different properties in different regimes of $\phi$ and $\theta$. In contrast to the conventional KT transition for surface roughening, HAGBs exhibit both static and dynamic critical-like behaviors. Similar critical behaviors have been observed in the shape fluctuations of biological membranes [51]. The unusual decreases in fluctuation amplitude and grain-boundary mobility after the roughening could be attributed to the widening of the grain boundary, which has not been considered in roughening theories. This conjecture needs to be further tested: For example, different quenching rates of premelted grain boundaries could result in different grain-boundary thicknesses, so their fluctuations can be compared. In addition, we perform phase field simulation (see SM for details) and confirm the experimental observations, including the nonmonotonic correlation length and time and $\phi_{R}$ approaching $\phi_{m}$ as $\theta$ decreases.

The mechanical, chemical, and electrical properties of crystals near the melting point are often different from those at low temperatures, which is commonly attributed to thermally excited defects [52]. In fact, grain-boundary roughening could play an important role since it removes flat facets and singularities associated with kinks, thus making the polycrystal more isotropic and uniform. Our results, especially the suppressed fluctuations after the roughening point, cast new light on the high-temperature behavior of crystals such as the abnormal decrease of the grain-boundary mobility [45] and the deviation of the free energy [53] near the melting point.

\section{ACKNOWLEDGMENTS}

We thank Tom Lubensky for helpful discussions and Wei $\mathrm{Li}$ for a supplementary video. This work was supported by RGC-GRF Grants No. 16301514, No. 16304015, and NSFC grant No. 111 Project (B16029).

The authors have no competing financial interests.

\section{APPENDIX A: CORRELATION FUNCTION AND STIFFNESS}

The length of a small section of the interface $\sqrt{d x^{2}+d y^{2}} \approx \sqrt{1+h^{\prime}(x)^{2}} d x \approx 1+h^{\prime}(x)^{2} / 2$, where $h^{\prime}=$ $\{[d h(x)] /(d x)\}=\tan \alpha \approx \alpha$ for a small-amplitude fluctuation. Consequently, the energy of an interface is

$$
\begin{aligned}
E & =b \int \gamma(\alpha) \sqrt{\mathrm{d} x^{2}+\mathrm{d} y^{2}} \\
& \approx b \int_{0}^{L}\left(\gamma+\gamma^{\prime} \alpha+\frac{\gamma^{\prime \prime}}{2} \alpha^{2}\right)\left(1+\frac{h^{\prime}(x)^{2}}{2}\right) \mathrm{d} x,
\end{aligned}
$$

where $\gamma^{\prime}=[(d \gamma) /(d \alpha)], L$ is the length of the measured grain boundary, $\alpha$ is the inclination angle, and $b$ is the width in the thin dimension, i.e., the $z$ direction as shown in Fig. 1. The integral of the first-order term about $h^{\prime}=$ $\tan \alpha \approx \alpha$ is 0 because the positive and negative $\alpha$ cancel each other out. Thus, the energy change relative to a flat interface is

$$
\Delta E=E-b L \gamma=\frac{1}{2} b \int_{0}^{L}\left(\gamma+\gamma^{\prime \prime}\right)\left(h^{\prime}\right)^{2} d x
$$

Here, $\Gamma \equiv \gamma+\gamma^{\prime \prime}$ is the stiffness [34].

Substituting the Fourier series $h(x)=\sum_{n=1}^{\infty}\left[h_{c}(k) \times\right.$ $\left.\cos (k x)+h_{S}(k) \sin (k x)\right]$ into Eq. (A2) yields $\Delta E=$ $\frac{1}{2} b\left(\gamma+\gamma^{\prime \prime}\right) L k^{2}|h(k)|^{2}$, where $k=2 n \pi / L$ and $|h(k)|^{2}=$ $h_{c}(k)^{2}+h_{s}(k)^{2}$ is the power spectrum. According to the equipartition theorem, each normal mode contributes to $k_{B} T / 2$ of energy, and thus $[25,34]$

$$
\left\langle|h(k)|^{2}\right\rangle=\frac{k_{B} T}{b L\left(\gamma+\gamma^{\prime \prime}\right)} \frac{1}{k^{2}} .
$$

The Fourier transformation of Eq. (A3) yields

$$
\begin{aligned}
g_{h}(x) & =\left\langle h\left(x_{0}\right) h\left(x_{0}+x\right)\right\rangle_{x_{0}}=\int_{0}^{\infty} h^{2}(k) \cos (k x) d k \\
& =\frac{k_{B} T}{2 \Gamma} \int_{0}^{\infty} \frac{1}{k^{2}+\xi^{-2}} \cos (k x) d k=\frac{k_{B} T}{4 \Gamma} \xi \exp (-x / \xi) .
\end{aligned}
$$

The correlation length $\xi$ is introduced in Eq. (A4) to avoid divergence as $k \rightarrow 0 \quad[19,25,54]$. Consequently, the normalized spatial correlation function is $g_{h}(x) / g_{h}(0)=$ $\exp (-x / \xi)$.

The Langevin equation of interface motion is $[34,44]$

$$
\frac{d h(x, t)}{d t}=M \Gamma \frac{d^{2} h(x, t)}{d x^{2}}+M \eta
$$

where the white noise $\eta$ satisfies $\left\langle\eta(x, t) \eta^{*}\left(x^{\prime}, t^{\prime}\right)\right\rangle \propto$ $\delta\left(x-x^{\prime}\right) \delta\left(t-t^{\prime}\right)$, and $M$ denotes mobility. The solution to Eq. (A5) is

$$
\begin{aligned}
h(k, t)= & h(k, 0) \exp \left(-M \Gamma k^{2} t\right) \\
& +M \exp \left(-M \Gamma k^{2} t\right) \int_{0}^{t} g(k, \tau) \exp \left(M \Gamma k^{2} \tau\right) d \tau,
\end{aligned}
$$

where $g$ is the Fourier transformation of $\eta$. Thus, 
(a)

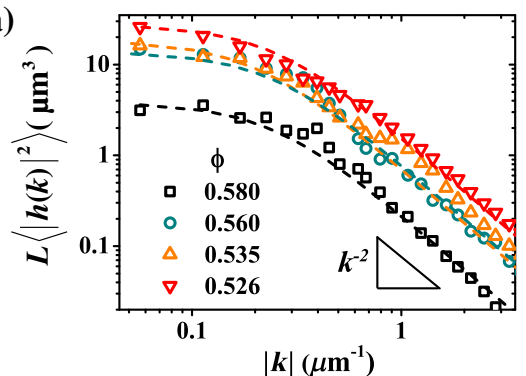

(d)

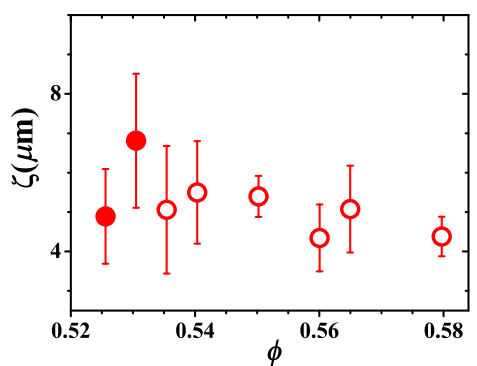

(b)

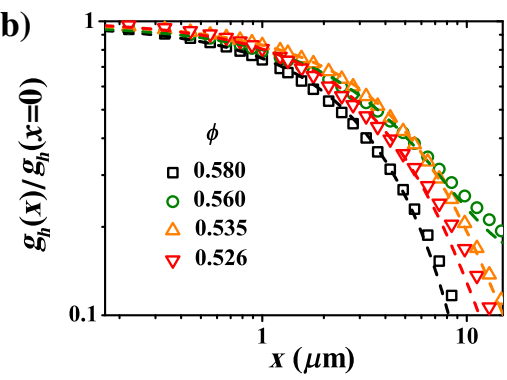

(e)

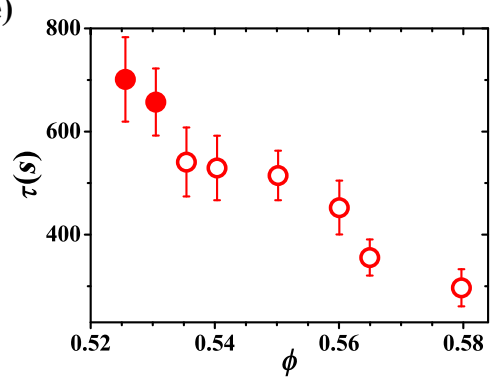

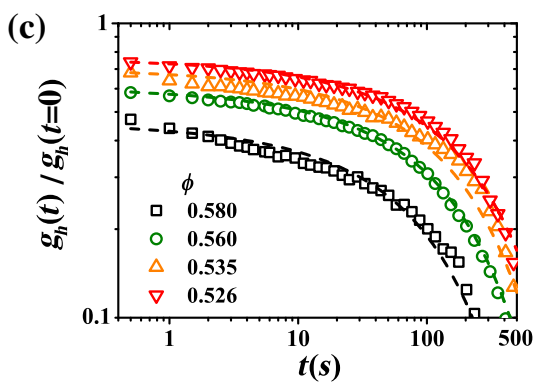

(f)

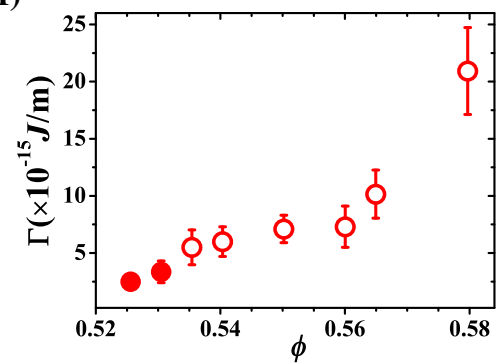

FIG. 6. Correlations and stiffness of the grain boundary with a mismatch angle $\theta=15^{\circ}$ at different volume fractions. (a) Powers spectra fitted with Eq. (A3). (b) The normalized spatial correlation $g_{h}(x) / g_{h}(x=0)$ fitted with $\exp (-x / \xi)$. (c) The normalized time correlation $g_{h}(t) / g_{h}(t=0)$ fitted with $\operatorname{erfc}(\sqrt{t / \tau})$. (d) The correlation length $\xi$ fitted from panel (b). (e) The correlation time $\tau$ fitted from panel (c). (f) The stiffness $\Gamma$ of the grain boundary. Filled symbols in panels (d)-(f) represent the properties of two solid-liquid interfaces of the melted grain boundary.

$$
\begin{aligned}
\left\langle h(k, t) h^{*}\left(k, t^{\prime}\right)\right\rangle & =\left\langle|h(k)|^{2}\right\rangle \exp \left[-M \Gamma k^{2}\left(t+t^{\prime}\right)\right]+M \exp \left[-M \Gamma k^{2}\left(t+t^{\prime}\right)\right] \int_{0}^{t} \int_{0}^{t}\left\langle g(k, \tau) g\left(k, \tau^{\prime}\right)\right\rangle \exp \left[M \Gamma k^{2}\left(\tau+\tau^{\prime}\right)\right] d \tau d \tau^{\prime} \\
& =\left\langle|h(k)|^{2}\right\rangle \exp \left[-M \Gamma k^{2}\left(t+t^{\prime}\right)\right]+\frac{C}{2 \Gamma k^{2}}\left(\exp \left[-M \Gamma k^{2}\left(t-t^{\prime}\right)\right]-\exp \left[-M \Gamma k^{2}\left(t+t^{\prime}\right)\right]\right) .
\end{aligned}
$$

When $t^{\prime}=0$,

$$
\left\langle h(k, t) h^{*}(k, 0)\right\rangle=\left\langle|h(k)|^{2}\right\rangle \exp \left(-M \Gamma k^{2} t\right) .
$$

The temporal correlation function is

$$
g_{h}(t)=\left\langle h\left(t_{0}\right) h\left(t_{0}+t\right)\right\rangle_{x_{0}, t_{0}} .
$$

Substituting Eq. (A8) into Eq. (A9) and integrating over $k$, we obtain

$$
\begin{aligned}
g_{h}(t) & =\frac{k_{B} T}{2 \pi\left(\gamma+\gamma^{\prime \prime}\right)} \int_{0}^{\infty} \frac{1}{k^{2}+\xi^{-2}} \exp \left(-M \Gamma k^{2} t\right) \mathrm{d} k \\
& =\frac{k_{B} T}{4 \Gamma} \operatorname{erfc}\left(\frac{\sqrt{M \Gamma t}}{\xi}\right),
\end{aligned}
$$

where erfc is the complementary error function. Thus, the normalized time correlation function $g_{h}(t) / g_{h}(0)=$ $\operatorname{erfc}\left[(t / \tau)^{1 / 2}\right]$, where the correlation time is defined as $\tau=\xi^{2} /(\Gamma M)$.

HAGBs exhibit similar power spectra and correlations as shown in Fig. 2 of the main text $\left(\theta=28^{\circ}\right)$ and Fig. 5 $\left(\theta=22^{\circ}\right)$. The fitted correlation length $\xi$ and time $\tau$ for HAGBs with $\theta=28^{\circ}$ [Figs. 2(d) and 2(e)], $\theta=22^{\circ}$ and LAGB with $\theta=15^{\circ}$ [Figs. 6(d) and 6(e)] show that the roughening point shifts toward the melting point as $\theta$ decreases and is preempted by melting at $\theta=15^{\circ}$.

\section{APPENDIX B: HEIGHT-DIFFERENCE CORRELATION FUNCTION}

Based on Eq. (A4), the height-difference correlation can be derived as

$$
\begin{aligned}
\left\langle(h(x+\Delta x, t+\Delta t)-h(x, t))^{2}\right\rangle & =\frac{1}{L} \int(h(x, t)-h(x+\Delta x, t+\Delta t))^{2} d x \\
& =\frac{k_{B} T}{\Gamma \pi} \int \frac{d k}{k^{2}+\xi^{-2}}\left[1-\cos (k \Delta x) \exp \left[-\eta\left(k^{2}+\xi^{-2}\right) \Delta t\right]\right] .
\end{aligned}
$$



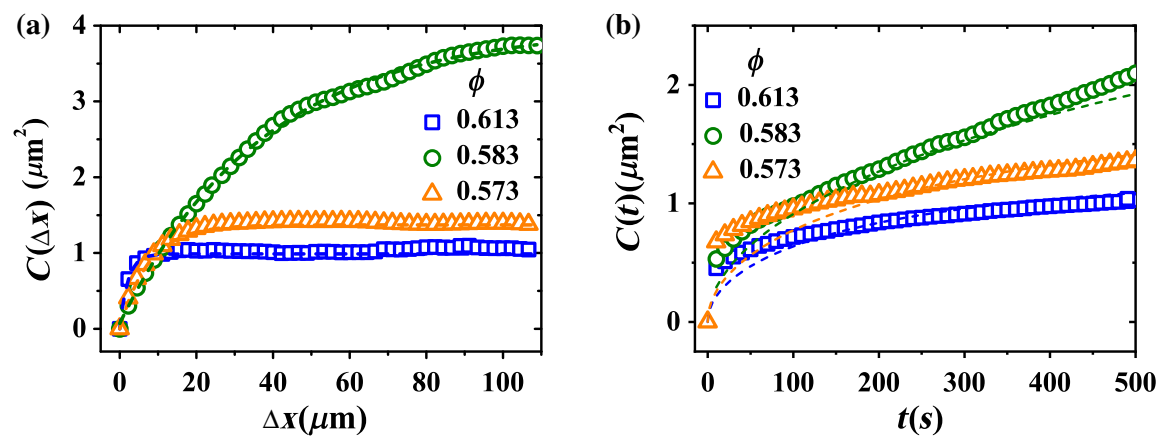

FIG. 7. (a) Spatial correlation function of the height difference fitted with Eq. (B2) (dashed curves). The fitted $\xi=2.47,37.6$, and $7.5 \mu \mathrm{m}$ at $\phi=0.613,0.583$, and 0.573 , respectively. (b) Time correlation function of the height difference fitted with Eq. (B3) (dashed curves). The fitted $\tau=402,2267$, and $657 \mathrm{~s}$ at $\phi=0.613,0.583$, and 0.573 , respectively.
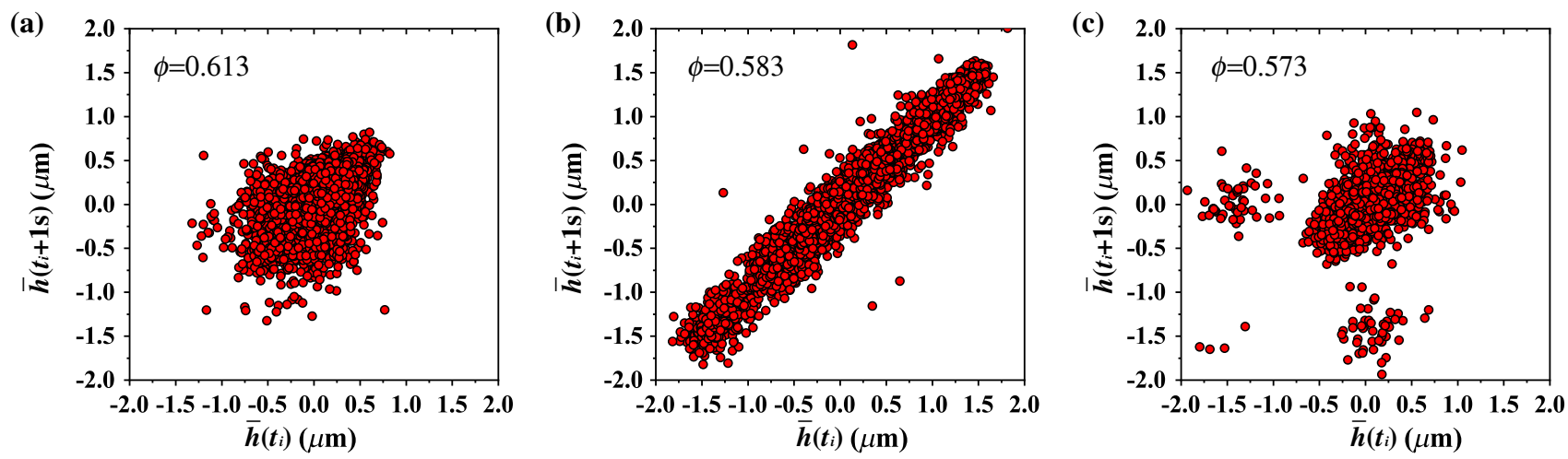

FIG. 8. Lag plots of $\bar{h}\left(t_{i}+1 \mathrm{~s}\right)$ versus $\bar{h}\left(t_{i}\right)$ for the grain boundary with $\theta=28^{\circ}$ at (a) $\phi=0.613$, (b) $\phi=0.583$, and (c) $\phi=0.573$.

Setting $\Delta t=0$ or $\Delta x=0$, we obtain

$$
\begin{aligned}
C(\Delta x) & =\left\langle(h(x+\Delta x, t)-h(x, t))^{2}\right\rangle \\
& =\frac{k_{B} T \xi}{2 \Gamma}[1-\exp (-\Delta x / \xi)], \\
C(\Delta t) & =\left\langle(h(x, t+\Delta t)-h(x, t))^{2}\right\rangle \\
& =\frac{k_{B} T \xi}{2 \Gamma} \operatorname{erf}\left((M \Gamma \Delta t)^{1 / 2} / \xi\right),
\end{aligned}
$$

where $\operatorname{erf}(x)$ denotes the error function. The correlation time $\tau=\xi^{2} /(M \Gamma)$. The height difference correlation functions and the fitted $\xi$ and $\tau$ in Fig. 7 agree well with the results in Figs. 2(d) and 2(e) of the main text.

Besides $g_{h}(t)$ in Fig. 2(c) of the main text, the temporal correlation can also be reflected by the lag plot of $\bar{h}\left(t_{i}+\Delta t\right)$ versus $\bar{h}\left(t_{i}\right)[55,56]$, where $\bar{h}$ is the center of mass of the grain boundary. If $\bar{h}\left(t_{i}+\Delta t\right)$ and $\bar{h}\left(t_{i}\right)$ are not correlated, then the lag plot is isotropic. Stronger correlations yield more anisotropic distributions. The lag plot in
Fig. 8 shows that the strongest correlation occurs near the roughening point $\phi=0.583$, featuring a high anisotropy.

\section{APPENDIX C: CORRELATION BETWEEN SOLID-LIQUID INTERFACES}

The correlation of the two solid-liquid interfaces of a melted grain boundary has rarely been studied in atomic or colloidal systems. It can be quantified by the crosscorrelation of their displacements,

$$
r(t)=\frac{\left\langle\left[h_{1}(x, t)-\bar{h}_{1}(t)\right]\left[h_{2}(x, t)-\bar{h}_{2}(t)\right]\right\rangle}{N \sigma_{1} \sigma_{2}}
$$

where $h_{i}(i=1,2)$ is the position of one solid-liquid interface with an average position $\bar{h}_{i}(t)$ and standard deviation $\sigma_{i}$, and $N$ is the number of discrete $x$ values. Each frame gives a value of $r$ within $[-1,1]$. Note that $r=1$ means that the two interfaces move in phase, while $r=-1$ means that they move in the opposite phase. The distribution in Fig. 9 shows that most $r>0$; hence, the two solid-liquid interfaces move with a positive correlation. 


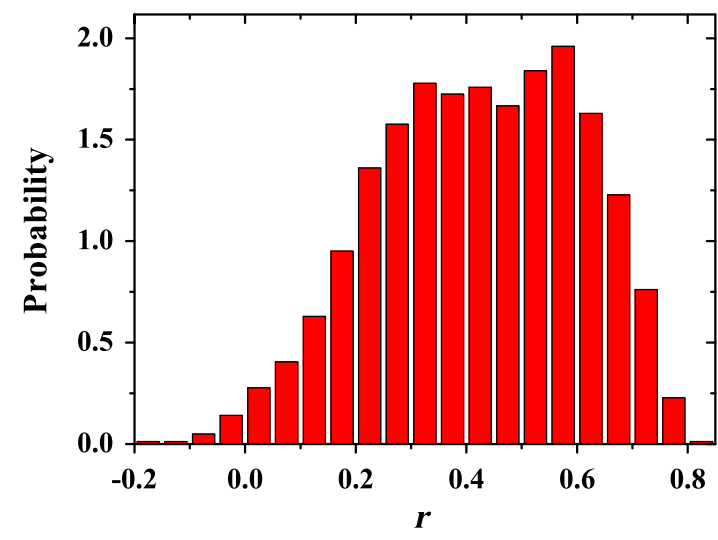

FIG. 9. Distribution of the correlation coefficient $r$ for the solidliquid interfaces of the melted $\theta=28^{\circ}$ grain boundary at $\phi=0.559$.

\section{APPENDIX D: ORIENTATION OF 5-7 PAIR}

We characterize the orientation of the grain boundary at the microscopic scale using the orientation of the dislocation, i.e., the pair of 5-neighbor and 7-neighbor particles. For LAGBs, the dislocations are disconnected, and thus their orientations can be clearly identified as along the grain boundary [see Fig. 10(a)]. By contrast, a HAGB is a connected long chain of 5-7-5-7-... particles without spaced-apart dislocations; hence, all particles are used twice for identifying dislocations except for the beginning and ending particles [Fig. 10(b)]. The orientations of 5-7 pairs are mainly along one of the [10] directions of a grain at $\phi>\phi_{R}$ for HAGBs [Fig. 10(f)]. When the system approaches the roughening point, the orientation distribution exhibits six sharp peaks [Fig. 10(g)], corresponding to the six [10] directions of the two grains. After the roughening at $\phi<\phi_{R}$, the peaks become broader because of the widening of the grain boundary. Consequently, the six sharp peaks of the orientation distribution could serve as a signature of the roughening.

\section{APPENDIX E: PHASE FIELD SIMULATION}

The phase field simulation is very effective for determining the evolution of the microstructure because it can resolve the density-wave structure of a polycrystalline material at the atomic scale while averaging out fluctuations $[39,57]$. We perform the phase field simulation of a monolayer polycrystal because the grain boundaries in our experiment are quasi-1D with a uniform shape in the $z$ direction. We describe the system by the free energy functional [57]

$$
\mathcal{F}=\int \mathrm{d}^{2} r\left[\psi / 2\left(u+\left(1+\nabla^{2}\right)^{2} \psi+\psi^{4} / 4\right)\right],
$$

where the order parameter $\psi$ is the density. Here, $\psi$ is relevant to the interaction between particles, but the relation (a)

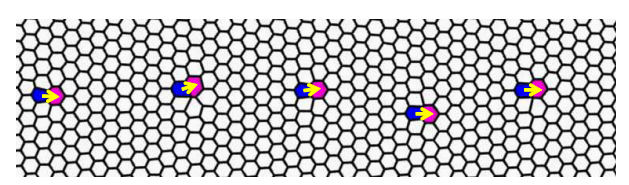

(c)
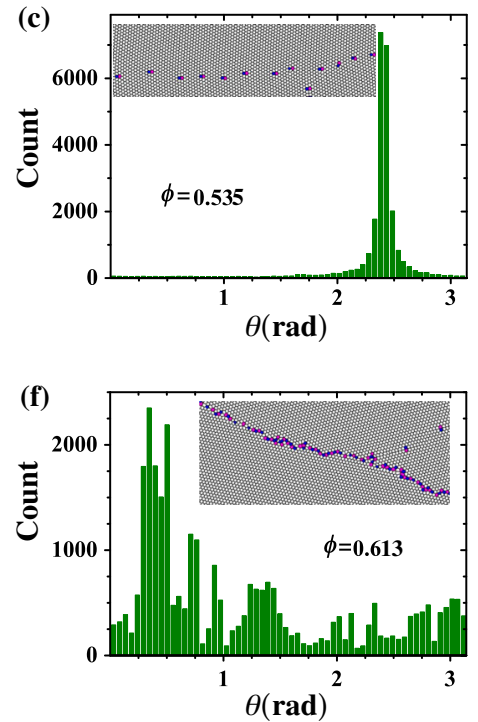
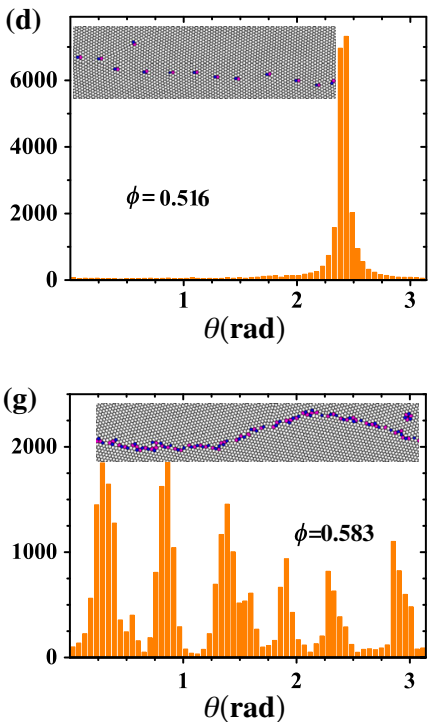

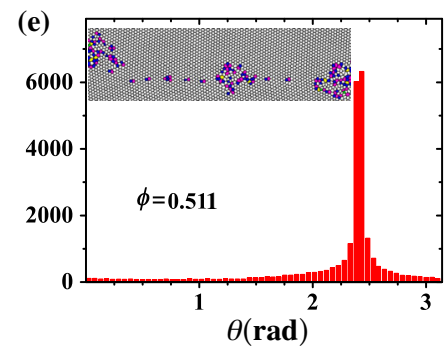

(b)
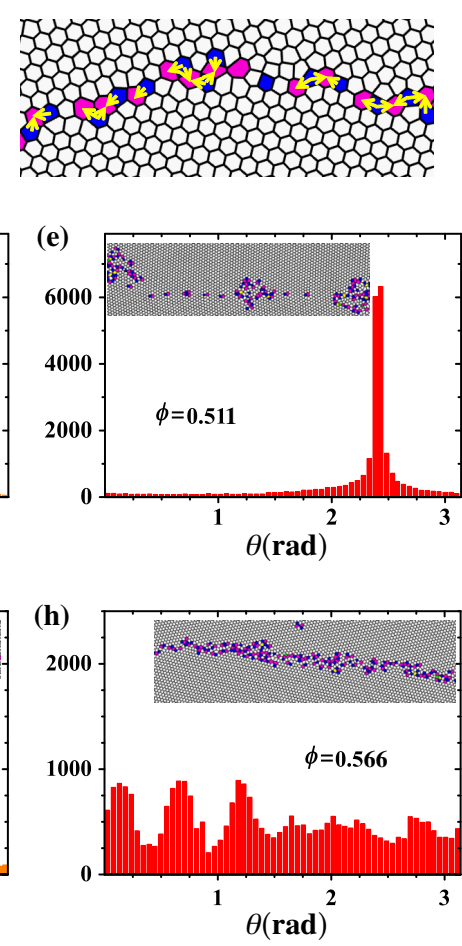

FIG. 10. (a,b) The Voronoi polygons with colored particles without exactly six nearest neighbors. A dislocation is a pair of 5- and 7-neighbor polygons; its orientation, defined as the direction from 5 to 7, is labeled with a yellow arrow. (a, c-e) The $\theta=8^{\circ}$ grain boundary. (b, $\mathrm{f}-\mathrm{h}$ ) The $\theta=28^{\circ}$ grain boundary. (c)-(h) Histograms of the orientations of 5-7 pairs in the grain boundaries. The statistics are calculated from 200 frames at each $\phi$. 
(a) $\phi=0.610$

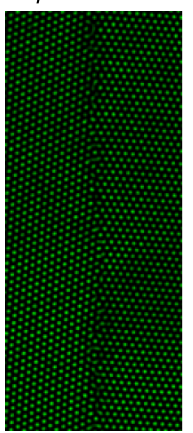

(b)

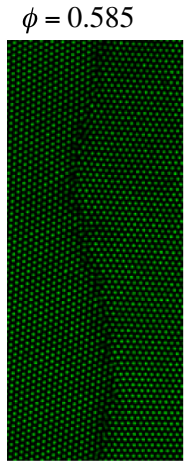

(d)
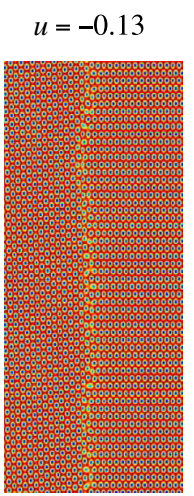

(e)

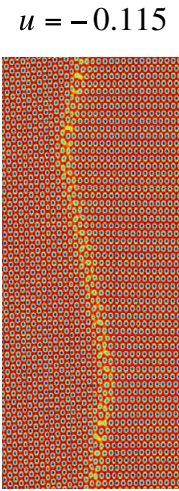

(c)

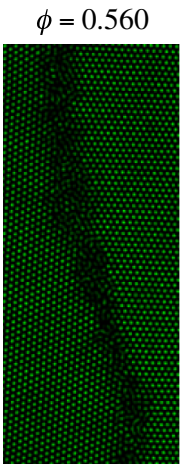

(f)

$u=-0.1$

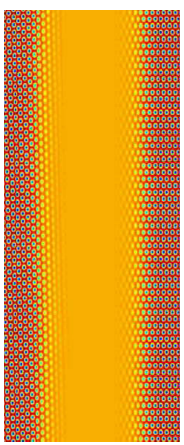

FIG. 11. (a)-(c) The grain boundary with $\theta=28^{\circ}$ in the experiment. These are subareas of Figs. 1(d), 1(f), and 1(h). (d)-(f) The grain boundary with $\theta=25.8^{\circ}$ at different effective temperatures $u$ in the phase field simulation.

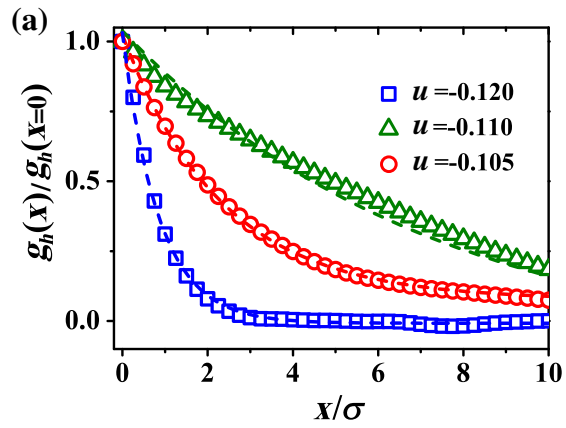

(c)

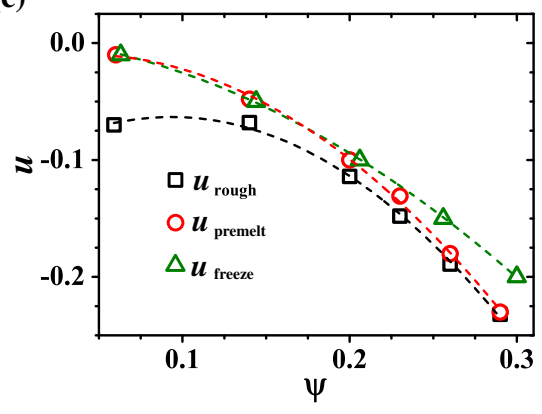

between $\phi$ and particle interaction is not available in the phase field simulation. Note that $u$ is the effective temperature or the chemical potential $\propto T-T_{\mathrm{m}} ; u<0$ for the solid phase, and $u>0$ for the liquid phase. The initial state contains two crystalline grains with a straight grain boundary at a given mismatch angle. It then evolves and equilibrates following the Cahn-Hilliard equation:

$$
\partial_{t} \psi=\Upsilon \frac{\delta F}{\delta \psi}+\eta
$$

by assuming the white noise $\eta$ to be 0 . Here, $\Upsilon$ is a phenomenological constant. The final equilibrium state gives the stable grain-boundary structure at a given temperature $u$. To further measure the grain-boundary fluctuations, we evolve the above equilibrium grain boundaries under Gaussian white noise $\eta$, which satisfies

$$
\left\langle\eta(\vec{r}, t) \eta\left(\vec{r}^{\prime}, t^{\prime}\right)\right\rangle=\Upsilon k_{B} T \nabla^{2} \delta\left(\vec{r}-\vec{r}^{\prime}\right) \delta\left(t-t^{\prime}\right)
$$

At a low temperature $(u=-0.13)$, the grain boundary is almost flat [Fig. 11(d)]. As the temperature increases to the roughening point $(u=-0.115)$, the grain boundary becomes rough and curved [Fig. 11(e)]. Further heating the system $(u=-0.1)$ causes the grain boundary to premelt [Fig. 11(f)]. These simulation results agree well with the corresponding experimental images in Figs. 11(a)-11(c).

The simulation shows that both the spatial and temporal correlations are the strongest near the roughening temperature $u_{R}$ and become weaker after the roughening (Fig. 12),

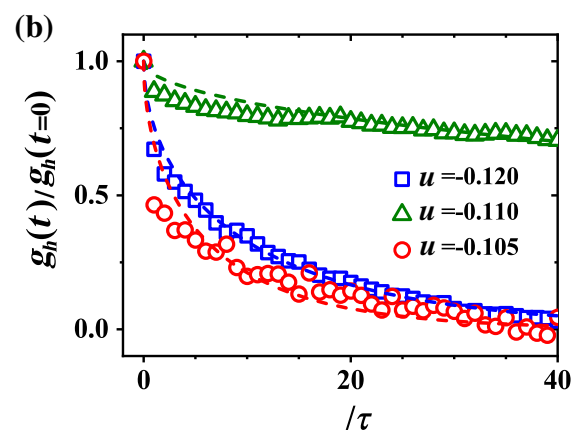

(d)

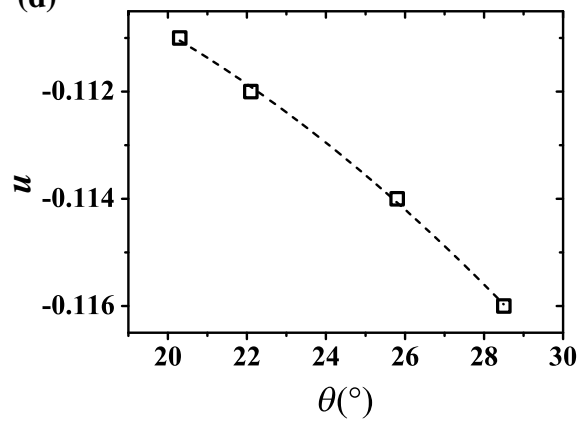

FIG. 12. Simulation results of the grain boundary with mismatch angle $\theta=25.8^{\circ}$, and initial inclination angles $\alpha_{1}=30^{\circ}$ and $\alpha_{2}=4.2^{\circ}$ at $\psi=0.2$. (a) Spatial correlation functions and (b) time correlation functions at different effective temperatures $u$. Note that $\sigma$ represents particle diameter and $\tau$ represents time step [57]. (c) The phase diagram with the roughening, premelting, and freezing curves. (d) The roughening point $u_{R}$ versus the mismatch angle. 
consistent with our experimental observation. Note that $u_{R}$ is identified from the slowest decay of $g_{h}(x)$ and $g_{h}(t)$ [Figs. 12(a) and 12(b)]. We also observe the onset of grain-boundary melting at $u_{\text {premelt }}$, and the solid phase melts completely at $u_{\text {melt }}$, as shown in Fig. 12(c). In addition, we perform simulations for different $\theta \mathrm{s}$ and find that $u_{R}$ decreases almost linearly with $\theta$, consistent with our experimental observation. Note that the particles' interactions in the experiments cannot be accurately modeled in the phase field simulations; hence, the simulation results cannot be compared directly with the experimental data. On the other hand, similar behaviors observed in the simulations and the experiments indicate that these properties are general and not sensitive to the exact form of particle interaction.

[1] G. Gottstein and L.S. Shvindlerman, Grain Boundary Migration in Metals: Thermodynamics, Kinetics, Applications (Taylor \& Francis Group, Boca Raton, 2009).

[2] D. Y. Yoon and Y. K. Cho, Roughening Transition of Grain Boundaries in Metals and Oxides, J. Mater. Sci. 40, 861 (2005).

[3] E. A. Holm and S. M. Foiles, How Grain Growth Stops: A Mechanism for Grain-Growth Stagnation in Pure Materials, Science 328, 1138 (2010).

[4] L. Lu, M. L. Sui, and K. Lu, Superplastic Extensibility of Nanocrystalline Copper at Room Temperature, Science 287, 1463 (2000).

[5] A. Rollett, F. J. Humphreys, G. S. Rohrer, and M. Hatherly, Recrystallization and Related Annealing Phenomena (Elsevier, New York, 2004).

[6] H. Hilgenkamp and J. Mannhart, Grain Boundaries in High-T $T_{c}$ Superconductors, Rev. Mod. Phys. 74, 485 (2002).

[7] H. Taub, G. Torzo, H. J. Lauter, and S. C. Fain, Jr., Phase Transitions in Surface Films 2, Vol. 267 (Springer Science \& Business Media, New York, 2012).

[8] S. T. Chui and J. D. Weeks, Dynamics of the Roughening Transition, Phys. Rev. Lett. 40, 733 (1978).

[9] J. D. Weeks and T. Riste, Ordering in Strongly Fluctuating Condensed Matter Systems (Plenum, New York, 1980).

[10] X.-Y. Liu, P. Bennema, and J. P. van der Eerden, RoughFlat-Rough Transition of Crystal Surfaces, Nature (London) 356, 778 (1992).

[11] M. S. Hoogeman, M. A. J. Klik, D. C. Schlößer, L. Kuipers, and J. W. M. Frenken, Real-Space Measurement of Surface Roughening, Phys. Rev. Lett. 82, 1728 (1999).

[12] H. P. Bonzel, 3D Equilibrium Crystal Shapes in the New Light of STM and AFM, Phys. Rep. 385, 1 (2003).

[13] S. T. Chui and J. D. Weeks, Phase Transition in the TwoDimensional Coulomb Gas, and the Interfacial Roughening Transition, Phys. Rev. B 14, 4978 (1976).

[14] H. van Beijeren, Exactly Solvable Model for the Roughening Transition of a Crystal Surface, Phys. Rev. Lett. 38, 993 (1977).

[15] S. T. Chui, Grain Boundary Roughening Transitions, Europhys. Lett. 87, 66001 (2009).

[16] D. L. Olmsted, E. A. Holm, and S. M. Foiles, Survey of Computed Grain Boundary Properties in Face-Centered
Cubic Metals II: Grain Boundary Mobility, Acta Mater. 57, 3704 (2009).

[17] K. G. F. Janssens, D. Olmsted, E. A. Holm, S. M. Foiles, S. J. Plimpton, and P. M. Derlet, Computing the Mobility of Grain Boundaries, Nat. Mater. 5, 124 (2006).

[18] H. Zhang, D. J. Srolovitz, J. F. Douglas, and J. A. Warren, Grain Boundaries Exhibit the Dynamics of Glass-Forming Liquids, Proc. Natl. Acad. Sci. U.S.A. 106, 7735 (2009).

[19] S. Gokhale, K. H. Nagamanasa, V. Santhosh, A. K. Sood, and R. Ganapathy, Directional Grain Growth from Anisotropic Kinetic Roughening of Grain Boundaries in Sheared Colloidal Crystals, Proc. Natl. Acad. Sci. U.S.A. 109, 20314 (2012).

[20] S. B. Lee and Y.-M. Kim, Kinetic Roughening of a $\sigma 5$ Tilt Grain Boundary in $\mathrm{SrTiO}_{3}$, Acta Mater. 57, 5264 (2009).

[21] D. T. J. Hurle, Handbook of Crystal Growth, Vol. 1 (NorthHolland, Amsterdam, 1993).

[22] C. Rottman, Roughening of Low-Angle Grain Boundaries, Phys. Rev. Lett. 57, 735 (1986).

[23] B. B. Straumal, V. N. Semenov, O. A. Kogtenkova, and T. Watanabe, Pokrovsky-Talapov Critical Behavior and Roughto-Rough Ridges of the $\sigma 3$ Coincidence Tilt Boundary in Mo, Phys. Rev. Lett. 92, 196101 (2004).

[24] B. Li, D. Zhou, and Y. Han, Assembly and Phase Transitions within Colloidal Crystals, Nat. Rev. Mater. 1, 15011 (2016).

[25] T. O. E. Skinner, D. G. A. L. Aarts, and R. P. A. Dullens, Grain-Boundary Fluctuations in Two-Dimensional Colloidal Crystals, Phys. Rev. Lett. 105, 168301 (2010).

[26] See Supplemental Material at http://link.aps.org/ supplemental/10.1103/PhysRevX.8.021045 for details on grain boundary identification and analysis.

[27] A. M. Alsayed, M. F. Islam, J. Zhang, P. J. Collings, and A. G. Yodh, Premelting at Defects within Bulk Colloidal Crystals, Science 309, 1207 (2005).

[28] Z. Wang, F. Wang, Y. Peng, Z. Zheng, and Y. Han, Imaging the Homogeneous Nucleation During the Melting of Superheated Colloidal Crystals, Science 338, 87 (2012).

[29] J. C. Crocker and D. G. Grier, Methods of Digital Video Microscopy for Colloidal Studies, J. Colloid Interface Sci. 179, 298 (1996).

[30] Z. T. Trautt and M. Upmanyu, Direct Two-Dimensional Calculations of Grain Boundary Stiffness, Scr. Mater. 52, 1175 (2005).

[31] J. Hernández-Guzmán and E. R. Weeks, The Equilibrium Intrinsic Crystal-Liquid Interface of Colloids, Proc. Natl. Acad. Sci. U.S.A. 106, 15198 (2009).

[32] V. D. Nguyen, M. T. Dang, B. Weber, Z. Hu, and P. Schall, Visualizing the Structural Solid-Liquid Transition at Colloidal Crystal/Fluid Interfaces, Adv. Mater. 23, 2716 (2011).

[33] D. G. A. L. Aarts, M. Schmidt, and H. N. W. Lekkerkerker, Direct Visual Observation of Thermal Capillary Waves, Science 304, 847 (2004).

[34] J. J. Hoyt, Z. T. Trautt, and M. Upmanyu, Fluctuations in Molecular Dynamics Simulations, Math. Comput. Simul. 80, 1382 (2010).

[35] P. M. Chaikin and T. C. Lubensky, Principles of Condensed Matter Physics (Cambridge University Press, Cambridge, England, 2000), Vol. 1. 
[36] R. H. Swendsen, Monte Carlo Studies of the Interface Roughening Transition, Phys. Rev. B 15, 5421 (1977).

[37] T. Emig and T. Nattermann, Roughening Transition of Interfaces in Disordered Systems, Phys. Rev. Lett. 81, 1469 (1998).

[38] K. K. Mon, D. P. Landau, and D. Stauffer, Interface Roughening in the Three-Dimensional Ising Model, Phys. Rev. B 42, 545 (1990).

[39] J. Mellenthin, A. Karma, and M. Plapp, Phase-Field Crystal Study of Grain-Boundary Premelting, Phys. Rev. B 78, 184110 (2008).

[40] P. R. Cantwell, M. Tang, S. J. Dillon, J. Luo, G. S. Rohrer, and M. P. Harmer, Grain Boundary Complexions, Acta Mater. 62, 1 (2014).

[41] Y. Peng, F. Wang, Z. Wang, A. M. Alsayed, Z. Zhang, A. G. Yodh, and Y. Han, Two-Step Nucleation Mechanism in Solid-Solid Phase Transitions, Nat. Mater. 14, 101 (2015).

[42] D. L. Olmsted, D. Buta, A. Adland, S. M. Foiles, M. Asta, and A. Karma, Dislocation-Pairing Transitions in Hot Grain Boundaries, Phys. Rev. Lett. 106, 046101 (2011).

[43] D. W. Demianczuk and K. T. Aust, Effect of Solute and Orientation on the Mobility of Near-Coincidence Tilt Boundaries in High-Purity Aluminum, Acta Metall. 23, 1149 (1975).

[44] Z. T. Trautt, M. Upmanyu, and A. Karma, Interface Mobility from Interface Random Walk, Science 314, 632 (2006).

[45] C. Deng and C. A. Schuh, Atomistic Simulation of Slow Grain Boundary Motion, Phys. Rev. Lett. 106, 045503 (2011).

[46] V. Privman, Fluctuating Interfaces, Surface Tension, and Capillary Waves: An Introduction, Int. J. Mod. Phys. C 03, 857 (1992).

[47] K. J. Strandburg, Two-Dimensional Melting, Rev. Mod. Phys. 60, 161 (1988).
[48] B. Li, F. Wang, D. Zhou, Y. Peng, R. Ni, and Y. Han, Modes of Surface Premelting in Colloidal Crystals Composed of Attractive Particles, Nature (London) 531, 485 (2016).

[49] Y. Peng, Z.-R. Wang, A. M. Alsayed, A. G. Yodh, and Y. Han, Melting of Multilayer Colloidal Crystals Confined between Two Walls, Phys. Rev. E 83, 011404 (2011).

[50] F. Wang, D. Zhou, and Y. Han, Melting of Colloidal Crystals, Adv. Funct. Mater. 26, 8903 (2016).

[51] A. R. Honerkamp-Smith, P. Cicuta, M. D. Collins, S. L. Veatch, M. den Nijs, M. Schick, and S. L. Keller, Line Tensions, Correlation Lengths, and Critical Exponents in Lipid Membranes near Critical Points, Biophys. J. 95, 236 (2008).

[52] W. D. Callister and D. G. Rethwisch, Materials Science and Engineering: An Introduction (Wiley, New York, 2007), Vol. 7.

[53] S M. Foiles, Temperature Dependence of Grain Boundary Free Energy and Elastic Constants, Scr. Mater. 62, 231 (2010).

[54] J. Hernández-Guzmán and E. R. Weeks, The Equilibrium Intrinsic Crystal-Liquid Interface of Colloids, Proc. Natl. Acad. Sci. U.S.A. 106, 15198 (2009).

[55] C. Mocuta, H. Reichert, K. Mecke, H. Dosch, and M. Drakopoulos, Scaling in the Time Domain: Universal Dynamics of Order Fluctuations in $\mathrm{Fe}_{3} \mathrm{Al}$, Science 308, 1287 (2005).

[56] M. Scheffer, S. R. Carpenter, T. M. Lenton, J. Bascompte, W. Brock, V. Dakos, J. van de Koppel, I. A. van de Leemput, S. A. Levin, E. H. Van Nes et al., Anticipating Critical Transitions, Science 338, 344 (2012).

[57] K. R. Elder and M. Grant, Modeling Elastic and Plastic Deformations in Nonequilibrium Processing Using Phase Field Crystals, Phys. Rev. E 70, 051605 (2004). 